\title{
Applying Positive Psychology Principles to Soccer Interventions for People with Mental Health Difficulties
}

\author{
Bettina Friedrichㅁ, Oliver J. Mason ${ }^{1,2}$ \\ ${ }^{1}$ Department of Clinical, Educational and Health Psychology, University College London, London, UK \\ ${ }^{2}$ School of Psychology, University of Surrey, Surrey, UK \\ Email: B.Friedrich@ucl.ac.uk,o.mason@surrey.ac.uk
}

How to cite this paper: Friedrich, B., \& Mason, O. J. (2018). Applying Positive Psychology Principles to Soccer Interventions for People with Mental Health Difficulties. Psychology, 9, 372-384. https://doi.org/10.4236/psych.2018.93023

Received: December 15, 2017

Accepted: March 25, 2018

Published: March 28, 2018

Copyright ( 2018 by authors and Scientific Research Publishing Inc. This work is licensed under the Creative Commons Attribution International License (CC BY 4.0).

http://creativecommons.org/licenses/by/4.0/

cc) (i) Open Access

\begin{abstract}
Adjunct exercise interventions for people with mental health difficulties have been shown to improve well-being while also increasing physical and social health. Soccer as a team sport is a particularly apt form of group-based exercise as it fosters social inclusion and communication skills potentially also across cultural and socio-economic barriers. We discuss how some exercise interventions such as those using soccer are potentially well-aligned with concepts from Positive Psychology such as Seligman's five elements (PERMA) that determine "Eudaimonia" (a good life): Positive emotions (P), Engagement and Flow (E), Positive Relationships (R), Meaning (M), and Accomplishment (A). In the present study the perceived life improvements reported by participants of a London-based soccer intervention "Coping Through Football" (CTF) are analysed for content using these five elements. All but Meaning (M) could be identified clearly; Positive Relationship (R) and Accomplishment (A) were the most commonly reported components. The PERMA model offers a potentially highly relevant framework to measure changes in well-being in participants of adjunct physical exercise treatments in mental health. Further quantitative and qualitative evaluation using the PERMA categories has the clear potential to inform policy and funding decisions in the growing area of psychosocial interventions in public mental health.
\end{abstract}

\section{Keywords}

Football, Soccer, Mental Health, Positive Psychology, Exercise

\section{Introduction}

Exercise interventions for people with mental health difficulties are very diverse 
(see Callaghan, 2004; Mason \& Holt, 2012a; Stathopoulou, Powers, Berry, Smits, \& Otto, 2006 for reviews) but include many that are aligned with the tenets of Positive Psychology as they focus on physical and mental well-being, social connectedness and community ties. While physical health is often a key objective, the importance of these interventions extends to psychological and social health. Physical exercise interventions have proven to be effective for participants with anxiety (Asmundson et al., 2013), schizophrenia (Faulkner \& Sparkes, 1999) and for the improvement of general mood (Barton, Griffin, \& Pretty, 2012). Fenton et al. (2017) have shown that the fostering of inclusion is a main mechanism by which exercise interventions contribute to increased well-being for people with mental health problems; this is not surprising as they often deal with issues of isolation (Brophy \& Harvey, 2011). Mason \& Holt (2012a) provide an interesting overview of outcomes of qualitative studies on physical activity interventions for people with mental health problems. Team sports may be particularly effective in enhancing psychosocial health through inclusion (Barber et al., 2001; Eime, Young, Harvey, Charity, \& Payne, 2013) and the positive direct impact of team sports on adolescent mental health has been shown by Steiner, McQuivey, Pavelski, Pitts, \& Kraemer (2000).

Among different team sports, soccer seems to hold a particular potential for increasing psychosocial health with a broad appeal to males in cultures that highly valorize this sport at least. Friedrich \& Mason (2017) provide an overview of the peer-reviewed literature on the effectiveness of soccer interventions for people with mental health problems. Evidence shows that these interventions are effective in increasing physical and mental well-being on a range of outcome measures and for a variety of target groups. Darongkamas et al. (2011) for example show in a mixed method study on men with mental health issues who took part in a soccer intervention, that participation improved mental health symptoms as well as attitudes about themselves, and general well. Carter-Morris \& Faulkner (2003) found that male service users experienced the soccer intervention as a meaningful opportunity for social interaction and those participants who were dealing with schizophrenia reported that participation challenged auditory hallucinations and delusional beliefs. Furthermore, Steckley (2005) showed in an observational study that soccer interventions improved inclusion, resilience and empowerment in boys is residential care who experience emotional and behavioral difficulties. Mason \& Holt (2012b) identified in a qualitative studies themes of recovery such as identifying with past self; service with a difference: opening up the social world; safety; empowerment; and feeling good. In a very recent study that used focus groups with participants, Lamont et al. (2017) have shown that participants in a walking football intervention in Scotland reported relational, personal and physical recovery-related benefits. Furthermore, soccer interventions could be shown to improve fitness (McElroy et al., 2008), general physical activity (Friedrich \& Mason, 2018), and physical fitness (O’Kane \& McKenna, 2002). 


\subsection{PERMA-Model: The Essential Elements of Well-Being}

In his book "Flourish", Seligman (2012) introduces a theory of human well-being as a multi-factorial construct. He states that "... no single measure defines it exhaustively (...), but several things contribute to it; these are the elements of well-being, and each of the elements is a measurable thing" (p. 15). According to Seligman, there are five elements that define well-being: Positive emotions (P), Engagement and Flow (E), Positive Relationships (R), Meaning (M), and Accomplishment (A). For a detailed description of these elements, see Seligman (2012: pp. 16-21). Seligman argues that these elements are defined by three properties: Firstly, they contribute to well-being. Secondly, they are pursued for their own sake and not with the purpose of gaining another element; and finally they are measured and defined independently of other elements.

Central to the theory is the assumption that true fulfilment (sometimes called "eudemonia") goes beyond mere pleasure, but also involves experienced meaningfulness and accomplishment. In their paper "Using well-being for public policy: Theory, measurement, and recommendations" Adler \& Seligman (2016: p. 5) state it thus: "Whereas hedonic well-being emphasizes the importance of feeling good, eudemonic well-being is characterized by functioning well in multiple domains of life". Hence an important and innovative implication of this model is that well-being cannot be measured along one single dimension, but rather that it is multi-dimensional, as true well-being is an experience that requires the satisfaction of a number of needs.

What contribution could this model make with regard to the evaluation (and application) of mental health interventions? By defining the key ingredients of true well-being, the model can help guide the development of interventions beyond mere symptom reduction. By operationalizing the components of well-being, these concepts may inform evaluation. In a comprehensive review Fenton et al. (2017: p. 12) concluded that "Both the recovery model and the tenets of positive psychology could be used more explicitly as theoretical frameworks to guide contemporary research regarding the potential of community-based recreation to influence recovery and social inclusion". A well-articulated and operationalized definition of well-being helps evaluation move beyond rather binary success/fail judgments by elucidating the relative strengths and weaknesses of an intervention's effects on the different key well-being dimensions. This information can be used to help improve and tailor intervention delivery to maximize benefits. However, empirical research explicitly using this model is limited to date. The following section examines how some of the findings of soccer-based interventions can be viewed within the PERMA model.

\subsection{Soccer Interventions through the PERMA Lens}

Soccer interventions as an adjunct treatment form to help people with mental health problems have a growing popularity (Friedrich \& Mason, 2017). A noteworthy feature of these interventions is their ability to benefit participants in a 
variety of ways. Most obviously there are physical health benefits (for a comparison of physical health benefits for different sports disciplines see Oja et al., 2015). Osborn (2001) has pointed out the need for improving physical health in people with psychiatric problems because of their high morbidity and the mortality rate. Studies have shown links between cardiovascular diseases and schizophrenia (Ringen, Engh, Birkenaes, Dieset, \& Andreassen, 2014) as well as with depression (Hare, Toukhsati, Johansson, \& Jaarsma, 2013), so the positive impact of soccer on cardiovascular function is very important (Krustrup et al., 2013; Krustrup et al., 2014). Boehm \& Kubzansky (2012) offer an interesting view on cardiovascular health, well-being and health behavior from a Positive Psychology perspective.

The benefits of soccer interventions go beyond physical health benefits. Due to its nature as team sports, soccer provides opportunity for social inclusion and can strengthen and rehabilitate social skills (Battaglia et al., 2013; Nielsen et al., 2014). A particular advantage of soccer interventions seems to be its attractiveness for people (especially men) across different ethnic and socio-demographic backgrounds (Mellor, 2008; Parnell \& Richardson, 2014). Yet another advantage is the relatively low costs and organizational demands of the activity. For these reasons, soccer has experienced a growing interest as intervention form, including in a therapeutic context; see for example Steckley (2005). Mutrie \& Faulkner (2004) made a very strong case for using Positive Psychology principles in exercise interventions for people with mental health problems concluding "we believe that physical activity participation epitomizes the principles of positive psychology" (p. 22). Lambert, D’Cruz, Schlatter, \& Barron (2016) took a similar stance and spoke out in favor of implementing the Positive Psychology approach for physical interventions in order to treat and prevent depression. Parschau et al. (2014) used the Positive Psychology approach to investigate the mechanisms in which physical exercise operate and conclude that experienced self-efficacy during exercise contributes largely to the positive experience and results in motivation for continuation of physical activity. Most recently, Van Cappellen, Rice, Catalino, \& Fredrickson (2017) have demonstrated the significance of positive affective processes during health related behavior in facilitating long-lasting positive health behavior change.

Many soccer interventions explicitly tackle several aspects of physical, psychological and social health that can be interpreted in terms of the PERMA elements (given in parentheses by ourselves). For example, a qualitative evaluation (Brawn, Combes, \& Ellis 2015) of the "Mental health and football well-being league" in the Northwest of England, suggested improvements across a range of elements in the participants: physical and psychological well-being (Enjoyment), sense of belonging (Positive Relationship), sense of achievement (Accomplishment) among others. Magee, Spaaij, \& Jeanes (2015) reported connectedness (Positive Relationship), counteracting stigma (Meaning), sense of safety (Enjoyment). As a last example, Butterly, Adams, Brown, \& Golby (2006) showed 
that a community based physical activity project including soccer led to the enjoyment of exercise (Enjoyment), increased self-confidence (Accomplishment), participants reported making more friends (Positive Relationship), participants planned educational and vocational qualifications (Meaning) and weight loss positively affected their self-esteem (Accomplishment). In addition to the spectrum of PERMA-relevant outcomes, even this short set of examples illustrates that different projects emphasize different components: one central issue for debate is whether competitiveness (Achievement) or team spirit (Positive Relations) should be emphasized (see for example Steckley, 2005). Similarly, participants often have varied expectations and experiences as to the benefits-some might be interested in improving their fitness and skills (Achievement), others might be more interested in the company, the sense of belonging (Positive Relationships) and the fun (Enjoyment). In conclusion, soccer interventions probably target many if not all of the PERMA-defined components of well-being, and have often proposed very similar themes to the elements of the model.

\subsection{The Intervention: Coping through Football (CTF)}

Coping Through Football (CTF) is a soccer scheme that has been developed as an adjunct treatment for people with mental health problems who are receiving secondary mental health treatment. This intervention is a collaboration between the London Playing Fields Foundation, the North East London Health Trust (NELFT) and the football club Leyton Orient. Participants attend informal soccer sessions regularly to improve their physical fitness and well-being, and to have the opportunity to socialize with other participants. Furthermore, healthy living workshops are organized as well as other social events such as soccer tournaments. Occupational therapists attend the sessions and monitor physical and emotional well-being of participants regularly; furthermore, they discuss exit routes and outcomes of the intervention with the participants and monitor their respective progress. This information is usually gathered at the start of the intervention and then collected bi-annually. Mason \& Holt (2012b) have previously carried out a qualitative evaluation of the intervention, and Friedrich \& Mason (2017) have reported quantitative outcomes. In this study we analyze further responses obtained from routine monitoring since 2012.

\section{Methods}

Between 2012-2017, 329 people (309 male, 97\%) participated in the Coping Through Football intervention. Participation was in principle open to anybody using the mental health services of the local NHS trust (NELFT)-health workers referred patients to the service when the soccer intervention seemed suitable and promising for their recovery. Attendance was completely voluntary and participants had the right to drop out any point without it affecting any other health services they are receiving through NELFT. The average age of the participants at the start date was 30.6 years $(\mathrm{SD}=10.08)$. The ethnic breakdown of this group 
is as follows: 122 white (37\%), 98 black (30\%), 59 Asian (18\%), 22 mixed (7\%), 15 other (5\%) and 13 unknown (4\%). Participants are asked to describe any changes noticed as a result of attendance at reviews that occur every six months (verbatim of the question: "What improvements to your life do you feel could be gained by taking part in the Coping Through Football Scheme?”). We included information on life improvement for participants who have been regularly attending the intervention over a period of at least one year and whose data was subsequently available for at least two time points. The number of times participants were reviewed depended on their length of participation at CTF; during some reviews these qualitative answers were not collected for pragmatic reasons such as time constraints. For the participants who could be included in this study, respective data on subjective life improvements was available on average from 2 - 3 reviews. Data was always taken from the most recent time point, leading to responses for 86 participants (78 male, 91\%). Average age of these participants was 30.11 years $(\mathrm{SD}=8.586)$. The ethnic breakdown of this group was 34 white (40\%), 27 black (31\%), 13 Asian (15\%), 11 mixed (13\%), and 1 unknown (1\%). Demographically, respondents were highly similar to attenders of the intervention as a whole. The breakdown of diagnoses was as follows: 36 participants had a psychosis (42\%), 25 emotional disorder(s) (29\%), 13 reported having drug and alcohol issues as main problem (15\%), 3 reported having a personality disorder (3\%), two participants had neurodevelopmental disorders (2\%); for 7 participants (8\%) the exact diagnoses was not known.

A content analysis was performed to classify statements according to the PERMA elements as defined by Seligman (2012) - the description of his model provided the coding system consisting of the five PERMA elements. The two authors reached an agreement as to how the PERMA elements were exactly to be interpreted and how the statements were to be assigned to these categories in the content analysis. The first coder conducted the content analysis and this was the thoroughly cross-checked by the second author. The categorization system was almost fully exhaustive-out of the 86 answers only one could not be assorted to a PERMA element, as practically all responses were related to one or more elements of well-being. The content analysis was carried out in close collaborations between the two authors. One author (BF) coded the statements which were then critically evaluated by the second author (OM). As the PERMA model clearly defines their elements, the assignments of the statements to categories used these definitions very explicitly and closely. When in doubt as to whether the item would truly fit into an element, a conservative approach was taken and the content was excluded. Some statements were assigned to more than just one category as their content applied to several elements of well-being.

\section{Results}

98.8\% of statements could be categorized using the coding scheme with $75.6 \%$ assigned to two or more categories. The categories are reported below in order of decreasing frequency. 


\subsection{Positive Relationship (R)}

The most commonly mentioned element was "Positive Relationship (R)": 58 out of $86(67 \%)$ gave responses that indicated that they felt participation led to positive relationship experiences. Interestingly not only positive relationships formed in the intervention sessions were mentioned ("coming to CTF has improved my life. I have made new friends only because I come here to CTF"; "CTF has provided me with opportunity to meet others that have experienced similar distressed"), but also relationships with people outside of the intervention ("I get on... much better with my family, my brother and we understand each other much better"). As evident from the last statement and the several other statements on positive relationships, the experience to connect to others was often interpreted by participants as indicative of improved communication skills and is therefore also experienced as an accomplishment. For some, increased self-esteem was also a part of this change ("CTF has improved my self-esteem-I have become more sociable and improved my interpersonal skills."). Additionally, relationship experiences seem to encourage some participants to reflect on own emotional competence ("Being with people. Need to think about what makes me angry. Need to think about dealing with anger", "Being around others in a social environment, semi-competitive environment is quite challenging-I am developing self-awareness"). Moreover, it helped combatting loneliness (" $\mathrm{I}]$ feel less lonely and [ $\mathrm{I}$ am] going out") and building a social support network ("My friends here have helped me out when I have had some troubles"). Furthermore, well-being was socially "infectious", perhaps pointing to a virtuous cycle ("I get happiness from seeing others happy").

The frequency and centrality of R-related statements underline the importance of the interpersonal side of team sports like soccer, and the positive impact of social contact very broadly ("going out, feeling better about myself"). Relationships were sustained outside of the intervention ("We chat on the phone between sessions").

\subsection{Achievement (A)}

The Achievement (A) element occurred second mostly commonly in 54 of 86 (63\%) responses. These accomplishments ranged from dealing with personal symptoms ("I was able to learn coping strategies and use them to overcome depressing and suicidal thoughts"); lifestyle choices ("II] reduce the amount of junk food I eat-I am eating more fruit and veg”); living arrangements (“II] was in secure hospital when I came to CTF, then I moved to support, now I am in independent flat"); improvements in personal interaction/relationships as mentioned above ("it has helped me with my confidence, made friends, engaging with a social life"); and achievements that were activity related ("I do feel good when I shoot a goal", "[CTF] made me feel more energetic [and] helped improve fitness"). 


\subsection{Engagement (E)}

Almost half the participants (40,47\%) reflected having benefitted from being able to engage in an activity in which they found satisfaction. As the respective statements often implied that they enjoyed the activity, these statements also contributed to "Positive Emotions (P)". Participants intrinsically appreciated engaging in the activity of playing soccer ("I like to play soccer for fun", "enjoyment of kicking the ball at CTF") and also commented on how the increase of fitness enhanced the enjoyment of this activity ("I get to enjoy playing fb more", "it [soccer] is something I love and I have got a natural talent"). Since many participants seem to have a very limited range of regular activities as a results of their mental health problem, the provision of an activity in which they could engage with is key ("it gets me out of the ward", "[CTF is] somewhere to go", "I wasn't doing much before CTF"), and has let to general activity ("II] have been going out more"), in particular engagements in other sports ("interested in gym, mixed martial arts"). It seems CTF has provided in some cases an exit route from a more sedentary, passive lifestyle and also provided structure ("[CTF] added structure").

\subsection{Positive Emotions (P)}

A similar number of participants $(38,44 \%)$ reported Positive Feelings (P). These were often expressed in connection with statements that touched on other elements of the PERMA model, for example when participants expressed pleasure of playing soccer and exercising ("enjoy soccer, scoring more goals", "getting a buzz from exercise", "I like the game, enjoy the match, I see my friends have a laugh"), the joy they get out of achievement ("My fitness has improved. I like scoring goals"; "Scoring goals-especially one, two [goals], involving two players") and the excitement of socializing with others ("I enjoy talking to the lads", "Meeting new people, new comers, forget my worries, makes me feel positive"). Hence the majority of these statements were also coding under other categories. General enjoyment was reported ("II] feel alive when I am at CTF"; "I relax, no stress", "challenging energy, endorphin release"; "CTF increases my well-being”), as well as reduced negative emotional states that might be associated with the mental health related symptoms ("Less anxious", "I feel no different to others. I am more myself, I am sure of myself. I am not angry"). Furthermore, over time a strengthened ability to feel enjoyment was perceived ("I enjoy soccer more").

\subsection{Meaning (M)}

Of all elements of the PERMA model, Meaning (M) was the one to which it was hardest to assigns comments to with a reasonable degree of certainty. The experience of a meaningful experience was not explicitly described but could sometimes indirectly be derived from the statements regarding life improvements. Due to the lack of certainty however, this category was felt too ambiguous to use in the present study. This may be a result of the very brief nature of feedback al- 
lowed. More in-depth analysis of longer qualitative interviews might reveal more insight into the perceived meaningfulness of the intervention.

\section{Discussion}

Despite some limitations discussed below, the PERMA model was very largely suitable for classifying reported life improvements in a meaningful and reasonably comprehensive manner for all statements relating to well-being. The fact that almost all statements could be categorized suggests a good fit between the content and the PERMA elements.

It is important to note that this project is an adjunct treatment intervention, with all participants having received secondary mental health care. This intervention is not intended nor designed to replace formal mental health treatment. Rather, it aims to add to the general well-being and resilience of the participants by providing a rewarding activity in a social context: the self-reported benefits of the intervention appear to be strongly consistent with the Positive Psychology approach-as defined in Seligman's well-being theory and respective PERMA model. The Coping Through Football intervention, in common with several soccer interventions, is explicitly aimed at increasing social inclusion: a psychosocial outcome indexed most closely by the Positive Relationship category. Thus it is encouraging that this was the most frequently endorsed life improvement by two-thirds of respondents. However, perceived improvements extended beyond this to encompass all but one of the PERMA categories. This is very encouraging for community public health interventions using sport/physical activity to engage users of mental health services.

A very large proportion of the perceived life improvements could be classified within the PERMA categories. Although not offering comprehensive coverage, the model provided a very useful framework for summarizing, re-presenting outcomes for a physical activity intervention in a mental health setting. The relative absence of Meaning statements could be due to the very brief nature of questioning here. Alternatively, Heintzelman \& King's (2014) have outlined several problems with the assumption that the pursuit of meaning is worthwhile for the individual. Category-based classification enabled a degree of quantification that, in another context, might have enabled analysis by demographics or other characteristics of the participants.

A further limitation is that the reported life improvements were not obtained in extensive in-depth qualitative interviews but rather from short answers collected in the context of regular health and well-being monitoring. For this reason, it is unlikely to be comprehensive in scope or depth, but instead captures the subjective, personal life improvement "highlights" prioritized by participants. Assigning content to the PERMA categories was necessarily somewhat subjective and the "Meaning (M)" element could not be reliably identified in the responses. Many statements could be, and indeed were, assigned to more than one PERMA element, as they reflected life improvements that touched on dif- 
ferent well-being components.

A recent review of the current literature on adjunct soccer interventions for people with mental health problems (Friedrich \& Mason, 2017) reported that most evaluations studies on soccer interventions use qualitative methods, with a relative dearth of quantitative evaluation studies. In part this may be due to a perceived lack of suitable outcome measures. Similar to Eime's approach (Eime et al., 2013), quantitative data pertaining to well-being could be used to develop measures tailored for physical activity. The PERMA model offers a sound basis for such a tool to measure impact in terms of its components of well-being.

The current study adds to the empirical evidence suggesting that soccer interventions show great potential as an effective adjunct intervention for people with mental health problems. Based on the present data, we are not able to draw reliable conclusions on the potential of other highly interactive team sports such as basketball for example to foster well-being as defined in the PERMA-model, but nothing speaks against the assumption that similar effects in fostering well-being in people with mental health problems might be found. Respective evaluation work could bring more light to this question. Greater evidence can help inform policy makers and potential funders as to approaches deserving of wider implementation alongside conventional medical and other treatment modalities. Positive Psychology offers an excellent framework in order to reshape conventional thinking in mental health.

\section{Conclusion}

In conclusion we believe that the aims of soccer interventions as adjunct treatment from are well aligned with principles and aims of the Positive Psychology approach. The outcomes of this study suggest that respective framework and methodology can be used to develop, implement and evaluate such interventions. Data for this study was limited both in the sense of number participants who provided information on subjective life improvements as well as the comprehensiveness of the available answers. Future evaluations might be able to use the Positive Psychology approach even more effectively by emphasizing qualitative data collection along the PERMA elements and allowing for more in depth responses. This might also provide more useful data with respect to the perceived meaningfulness of the intervention. The present study suggests that there is a great potential in using Positive Psychology as a framework for understanding mechanisms and effectiveness of soccer interventions, and possible of sports interventions in general.

\section{Acknowledgements}

This research was funded by the NIHR School for Public Health Research (SPHR) Public Health Practice Evaluation Scheme (PHPES). The views expressed are those of the author(s) and not necessarily those of the NHS, the NIHR or the Department of Health. The NIHR School for Public Health Re- 
search (SPHR) is a partnership between the Universities of Sheffield, Bristol, Cambridge, Imperial College London, UCL; The London School for Hygiene and Tropical Medicine; the LiLaC collaboration between the Universities of Liverpool and Lancaster and Fuse; The Centre for Translational Research in Public Health, a collaboration between Newcastle, Durham, Northumbria, Sunderland and Teesside Universities. Grant holder is Dr Oliver Mason. We thank the staff and participants of the intervention, and particularly Sonia Smith, Andrea Shepherd and Barbara Armstrong for their invaluable role in its evaluation.

\section{References}

Adler, A., \& Seligman, M. E. (2016). Using Well-Being for Public Policy: Theory, Measurement, and Recommendations. International Journal of Well-being, 6, 1-35. https://doi.org/10.5502/ijw.v6i1.429

Asmundson, G. J., Fetzner, M. G., DeBoer, L. B., Powers, M. B., Otto, M. W., \& Smits, J. A. (2013). Let's get Physical: A Contemporary Review of the Anxiolytic Effects of Exercise for Anxiety and Its Disorders. Depression and Anxiety, 30, 362-373. https://doi.org/10.1002/da.22043

Barber, B. L., Eccles, J. S., \& Stone, M. R. (2001). Whatever Happened to the Jock, the Brain, and the Princess? Young Adult Pathways Linked to Adolescent Activity Involvement and Social Identity. Journal of Adolescent Research, 16, 429-455. https://doi.org/10.1177/0743558401165002

Barton, J., Griffin, M., \& Pretty, J. (2012). Exercise-, Nature- and Socially Interactive-Based Initiatives Improve Mood and Self-Esteem in the Clinical Population. Perspectives in Public Health, 132, 89-96. https://doi.org/10.1177/1757913910393862

Battaglia, G., Alesi, M., Inguglia, M., Roccella, M., Caramazza, G., Bellafiore, M., \& Palma, A. (2013). Soccer Practice as an Add-On Treatment in the Management of Individuals with a Diagnosis of Schizophrenia. Neuropsychiatric Disease and Treatment, 9, 595-603. https://doi.org/10.2147/NDT.S44066

Boehm, J. K., \& Kubzansky, L. D. (2012). The Heart's Content: The Association between Positive Psychological Well-Being and Cardiovascular Health. Psychological Bulletin, 138, 655-691. https://doi.org/10.1037/a0027448

Brawn, P., Combes, H., \& Ellis, N. (2015). Football Narratives: Recovery and Mental Health. The Journal of New Writing in Health and Social Care, 2, 30-46.

Brophy, L., \& Harvey, C. (2011). Social Isolation in People with Mental Illness. Medicine Today, 12, 73-78.

Butterly, R., Adams, D., Brown, A., \& Golby, J. (2006). Client Perceptions of the MUSCSEL Project: A Community-Based Physical Activity Programme for Patients with Mental Health Problems. Journal of Public Mental Health, 5, 45-52. https://doi.org/10.1108/17465729200600031

Callaghan, P. (2004). Exercise: A Neglected Intervention in Mental Health Care? Journal of Psychiatric and Mental Health Nursing, 11, 476-483.

Carter-Morris, P., \& Faulkner, G. (2003). A Football Project for Service Users: The Role of Football in Reducing Social Exclusion. Journal of Mental Health Promotion, 2, 24-30.

Darongkamas, J., Scott, H., \& Taylor, E. (2011). Kick-Starting Men’s Mental Health: An Evaluation of the Effect of Playing Football on Mental Health Service Users' Well-Being. International Journal of Mental Health Promotion, 13, 14-21. 
https://doi.org/10.1080/14623730.2011.9715658

Eime, R. M., Young, J. A., Harvey, J. T., Charity, M. J., \& Payne, W. R. (2013). A Systematic Review of the Psychological and Social Benefits of Participation in Sport for Children and Adolescents: Informing Development of a Conceptual Model of Health through Sport. International Journal of Behavioral Nutrition and Physical Activity, 10, 98.

Faulkner, G., \& Sparkes, A. (1999). Exercise as Therapy for Schizophrenia: An Ethnographic Study. Journal of Sport and Exercise Psychology, 21, 52-69. https://doi.org/10.1123/jsep.21.1.52

Fenton, L., White, C., Gallant, K. A., Gilbert, R., Hutchinson, S., Hamilton-Hinch, B., \& Lauckner, H. (2017). The Benefits of Recreation for the Recovery and Social Inclusion of Individuals with Mental Illness: An Integrative Review. Leisure Sciences, 39, 1-19. https://doi.org/10.1080/01490400.2015.1120168

Friedrich, B., \& Mason, O. J. (2017). "What Is the Score?" A Review of Football-Based Public Mental Health Interventions. Journal of Public Mental Health, 16, 144-158. https://doi.org/10.1108/JPMH-03-2017-0011

Friedrich, B., \& Mason, O. J. (2018). Evaluation of the Coping through Football Project: Physical Activity and Psychosocial Outcomes. The Open Public Health Journal, 10, 276-282.

Hare, D. L., Toukhsati, S. R., Johansson, P., \& Jaarsma, T. (2013). Depression and Cardiovascular Disease: A Clinical Review. European Heart Journal, 35, 1365-1372. https://doi.org/10.1093/eurheartj/eht462

Heintzelman, S. J., \& King, L. A. (2014). Life Is Pretty Meaningful. American Psychologist, 69, 561-574. https://doi.org/10.1037/a0035049

Krustrup, P., Hansen, P. R., Nielsen, C. M., Larsen, M. N., Randers, M. B., Manniche, V. et al. (2014). Structural and Functional Cardiac Adaptations to a 10-Week School-Based Football Intervention for 9-10 Year-Old Children. Scandinavian Journal of Medicine \& Science in Sports, 24, 4-9. https://doi.org/10.1111/sms.12277

Krustrup, P., Randers, M. B., Andersen, L. J., Jackman, S. R., Bangsbo, J., \& Hansen, P. R. (2013). Soccer Improves Fitness and Attenuates Cardiovascular Risk Factors in Hypertensive Men. Medicine and Science in Sports and Exercise, 45, 553-560. https://doi.org/10.1249/MSS.0b013e3182777051

Lambert, L., D’Cruz, A., Schlatter, M., \& Barron, F. (2016). Using Physical Activity to Tackle Depression: The Neglected Positive Psychology Intervention. Middle East Journal of Positive Psychology, 2, 42-60.

Lamont, E., Harris, J., McDonald, G., Kerin, T., \& Dickens, G. L. (2017). Qualitative Investigation of the Role of Collaborative Football and Walking Football Groups in Mental Health Recovery. Mental Health and Physical Activity, 12, 116-123. https://doi.org/10.1016/j.mhpa.2017.03.003

Magee, J., Spaaij, R., \& Jeanes, R. (2015). “It's Recovery United for Me”: Promises and Pitfalls of Football as Part of Mental Health Recovery. Sociology of Sport Journal, 32, 357-376. https://doi.org/10.1123/ssj.2014-0149

Mason, O. J., \& Holt, R. (2012a). Mental Health and Physical Activity Interventions: A Review of the Qualitative Literature. Journal of Mental Health, 21, 274-284. https://doi.org/10.3109/09638237.2011.648344

Mason, O. J., \& Holt, R. (2012b). A Role for Football in Mental Health: The Coping through Football Project. The Psychiatrist, 36, 290-293.

https://doi.org/10.1192/pb.bp.111.036269 
McElroy, P., Evans, P., \& Pringle, A. (2008). Sick as a Parrot or over the Moon: An Evaluation of the Impact of Playing Regular Matches in a Football League on Mental Health Service Users. Practice Development in Health Care, 7, 40-48. https://doi.org/10.1002/pdh.245

Mellor, G. (2008). The Janus-Faced Sport: English Football, Community and the Legacy of the "Third Way". Soccer \& Society, 9, 313-324. https://doi.org/10.1080/14660970802008942

Mutrie, N. and Faulkner, G. (2004) Physical Activity: Positive Psychology in Motion. In P. A. Linley, \& S. Joseph (Eds.), Positive Psychology in Practice (pp. 146-164). Hoboken, NJ: John Wiley \& Sons, Inc. https://doi.org/10.1002/9780470939338.ch9

Nielsen, G., Wikman, J. M., Jensen, C. J., Schmidt, J. F., Gliemann, L., \& Andersen, T. R. (2014). Health Promotion: The Impact of Beliefs of Health Benefits, Social Relations and Enjoyment on Exercise Continuation. Scandinavian Journal of Medicine \& Science in Sports, 24, 66-75. https://doi.org/10.1111/sms.12275

O’Kane, P., \& McKenna, B. (2002). Five a Side Makes a Difference. Mental Health Nursing, 22, 6-9.

Oja, P., Titze, S., Kokko, S., Kujala, U. M., Heinonen, A., Kelly, P. et al. (2015). Health Benefits of Different Sport Disciplines for Adults: Systematic Review of Observational and Intervention Studies with Meta-Analysis. British Journal of Sports Medicine, 49, 434-440. https://doi.org/10.1136/bjsports-2014-093885

Osborn, D. P. (2001). The Poor Physical Health of People with Mental Illness. Western Journal of Medicine, 175, 329-332. https://doi.org/10.1136/ewjm.175.5.329

Parnell, D., \& Richardson, D. (2014). Introduction. Soccer \& Society, 15, 823-827. https://doi.org/10.1080/14660970.2014.920619

Parschau, L., Fleig, L., Warner, L. M., Pomp, S., Barz, M., Knoll, N. et al. (2014). Positive Exercise Experience Facilitates Behavior Change via Self-Efficacy. Health Education \& Behavior, 41, 414-422. https://doi.org/10.1177/1090198114529132

Ringen, P. A., Engh, J. A., Birkenaes, A. B., Dieset, I., \& Andreassen, O. A. (2014). Increased Mortality in Schizophrenia Due to Cardiovascular Disease-A Non-Systematic Review of Epidemiology, Possible Causes, and Interventions. Frontiers in Psychiatry, 5, 137. https://doi.org/10.3389/fpsyt.2014.00137

Seligman, M. E. (2012). Flourish: A Visionary New Understanding of Happiness and Well-Being: Simon and Schuster. New York, NY: Atria Books.

Stathopoulou, G., Powers, M. B., Berry, A. C., Smits, J. A., \& Otto, M. W. (2006). Exercise Interventions for Mental Health: A Quantitative and Qualitative Review. Clinical Psychology: Science and Practice, 13, 179-193.

Steckley, L. (2005). Just a Game? The Therapeutic Potential of Football Facing Forward: Residential Child Care in the 21st Century (pp. 137-147). Lyme Regis: Russell House Publishing.

Steiner, H., McQuivey, R. W., Pavelski, R., Pitts, T., \& Kraemer, H. (2000). Adolescents and Sports: Risk or Benefit? Clinical Pediatrics, 39, 161-166. https://doi.org/10.1177/000992280003900304

Van Cappellen, P., Rice, E. L., Catalino, L. I., \& Fredrickson, B. L. (2017). Positive Affective Processes Underlie Positive Health Behaviour Change. Psychology \& Health, 33, 77-97. 